\title{
Recent Developments in Stellar Pulsation Theory
}

\author{
A. Gautschy \\ Astronomisches Institut der Universität Basel, Venusstr. 7, CH-4102 \\ Binningen, Switzerland
}

\begin{abstract}
A major driving force behind the efforts invested in stellar pulsation calculations in the recent years was certainly the success story of the new generation of opacity data. The new opacities did not only solve old problems but they also increased the complexity of quantitative stellar pulsation studies. Besides surveying the consequences of the new generation of stellar opacities for pulsation theory we also address more formal aspects of dealing with stellar oscillations. The section reviewing recent literature on the application of stellar pulsation theory to different classes of pulsating variables is strongly biased towards the high-luminosity part of the Hertzsprung-Russell (HR) diagram. We finish by mentioning recent changes in our beliefs concerning the envelope structure of oscillating white dwarfs.
\end{abstract}

\section{Introduction}

Computing stellar pulsation problems was considerably revived in recent years after the release of a new generation of stellar opacity data. The dramatic increase of the Rosseland opacity mean in the range of a few hundred thousand Kelvin allowed outstanding problems in stellar pulsation theory to be solved. But not only old problems were revisited with new microphysical data and mature computer codes also new features, that are to be attributed to the improved opacities, in the pulsational behaviour of stars were encountered.

The Hertzsprung-Russell diagram in Fig. 1 shows the distribution of a small subset of all pulsating variables. Essentially all major evolutionary stages over a large range of stellar masses encounter pulsational instabilities. Hence, pulsations may be potentially important tools for probing aspects of stellar constitution over a wide range of the physical parameter space. Recently, Brown et al. (1994) addressed the prospected gain in accuracy to constrain global stellar quantities - e.g., mass, luminosity, chemical abundances, age - from solar-like oscillations in distant stars. The results were rather disillusioning, however, in particular when compared with the achievements on the sun. The main reason for the reduced information gain is obviously the constraint of whole-disk observations only in distant stars.

Nevertheless, high-quality stellar oscillation data can play a role in a new era of quantitative stellar physics. Observational efforts and instrumental developments reached a level that allow the detection and the monitoring of variability at amplitudes of the order of $10^{-3}$ mag as a matter of routine. This achieve- 


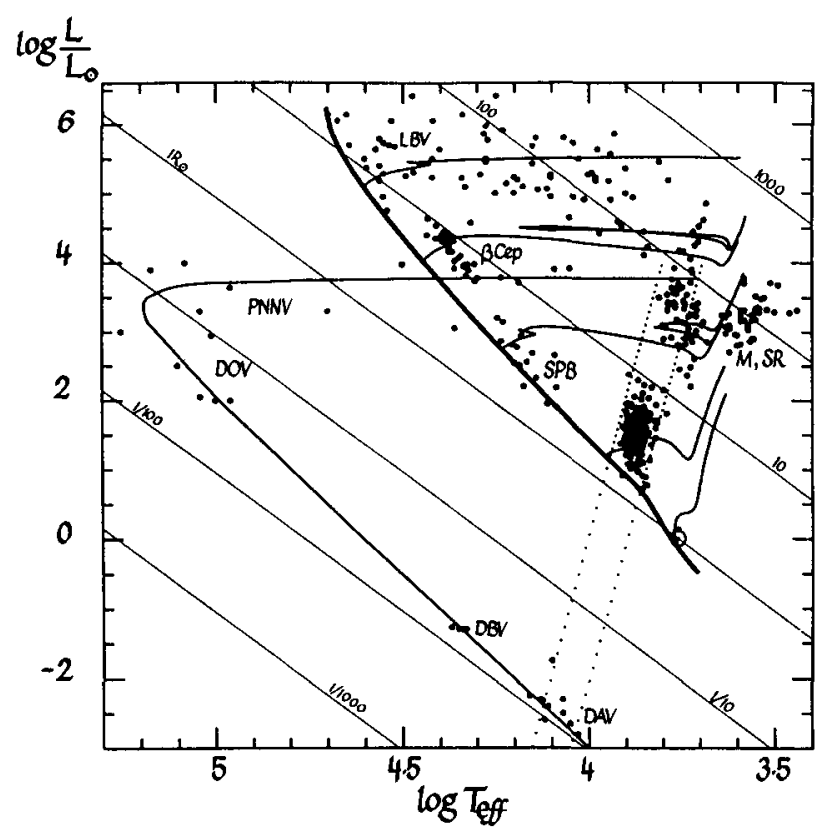

Figure 1. Distribution of some observed pulsating variable stars (dots) on the HR diagram. For convenience some guiding lines from stellar evolution are included. The heavy line passing from upper left to lower right shows the ZAMS. Evolutionary paths for some stellar masses, labeled at the tracks, are included. Common abbreviations are added to the figure indicating the location of some major families pulsating variable stars.

ment went along with the identification of a multitude of modes in stars that were believed to be mono-mode pulsators or even stable stars hitherto. Therefore, future observational approaches to stellar pulsations will conceivably shift from monitoring secular changes to long time-series monitoring of multi-mode pulsational variability - most of them typically on very low amplitude levels.

The following review must be considered as a biased survey of the recent literature on the theory of stellar pulsations. Particular emphasis is put on the impact of the new generation of opacity data, on pulsational instabilities in luminous stars, and on recent challenges of our beliefs concerning white-dwarf envelopes.

\section{Methodical aspects of stellar pulsation theory}

Many of the results in the more formal topics of stellar pulsation theory were driven by the requirement to interpret the high-quality data from the solar oscillation monitoring. In particular, second-order expansions for the coupling of nonradial oscillations with stellar rotation and a possible magnetic field were derived (Gough \& Thompson 1990, Dziembowski \& Goode 1992, Goode \& Thomp- 
son 1992). Takata \& Shibahashi (1994) suggested using directly vector spherical harmonics for an easier derivation of the selection rules for perturbations to nonradial eigenfunctions in rotating, magnetized stars. They aimed at explaining the observed frequency splittings in roAp stars, in particular the septuplet claimed for HR3831 (Kurtz et al. 1993).

Pulsation - convection coupling still defies a satisfactory amalgamation. Motivated by solar oscillations, Balmforth $(1992 \mathrm{a}$, b) discussed comprehensively the effects on radial oscillation modes in the sun of his time-dependent, non-local mixing-length approach. In the third paper of the series, Balmforth (1992c) criticizes the lack of predictive power of the excitation theories of solar modes based on stochastic driving. Xiong (1991, and references therein) is an advocate of a statistical approach to convection. He proposed a scheme for pulsation - convection coupling, but only the troublesome local convection theory found some applications to stars (Xiong 1981). Stellingwerf, on the other hand applied his non-local, time-dependent approach extensively to RR Lyrae stars (Stellingwerf 1984 , and references therein).

Despite the powerful and user-friendly computers presently available, we are still far from being able to simulate nonlinear, nonradial oscillations of stars. An alternative approach is the use of nonlinear mode coupling equations. In this approach, the temporal evolution of the amplitudes of a preselected set of modes is described by a system of nonlinearly coupled equations. The coefficients in the equations are typically a combination of linear eigenmodes and of stellar structure quantities. Very recently, Goupil \& Buchler (1994) and Van Hoolst (1994) presented the first, rather intricate, derivations of nonlinear, nonadiabatic, nonradial amplitude equations. The complexity of the problem is considerable and we eagerly await the first predictions for frequency splittings and amplitude distributions of multiplet components due to nonlinear interaction.

Results from adaptive-mesh radiation hydrodynamic codes were published in the last few years. Gehmeyr $(1992,1993)$ studied RR Lyrae stars including a time-dependent convection treatment which is not far from the Stellingwerf approach. Feuchtinger \& Dorfi (1994) presented very smooth lightcurves for purely radiative RR Lyare star models. In both approaches, it seems that a fully adaptive mesh is not applicable due to numerical error propagation, in particular due to those picked up in the deep interior if the mesh is moving too unconstrained and which are then advected outwards. Hence, the mesh is freely adaptive in the exterior only, close to the driving region but it remains Lagrangian in the deep interior. Such a treatment is reminiscent of the Castor et al. (1977) method.

Also in the neutron star community, stellar stability problems led to innovative developments. To investigate sectorial oscillation modes in rapidly rotating neutron star models (in the Newtonian limit), Ipser \& Lindblom (1990) made use of and adapted a two-potential approach to hydrodynamics that was originally proposed by Poincaré. First-order post-Newtonian corrections to the neutron star oscillation frequencies were provided by Cutler (1991) and Cutler \& Lindblom (1992). Kokkotas \& Schutz (1992) discussed the mathematical aspects of quasi-normal modes that occur when not only the fluid configuration but also the space-time metric is perturbed. 


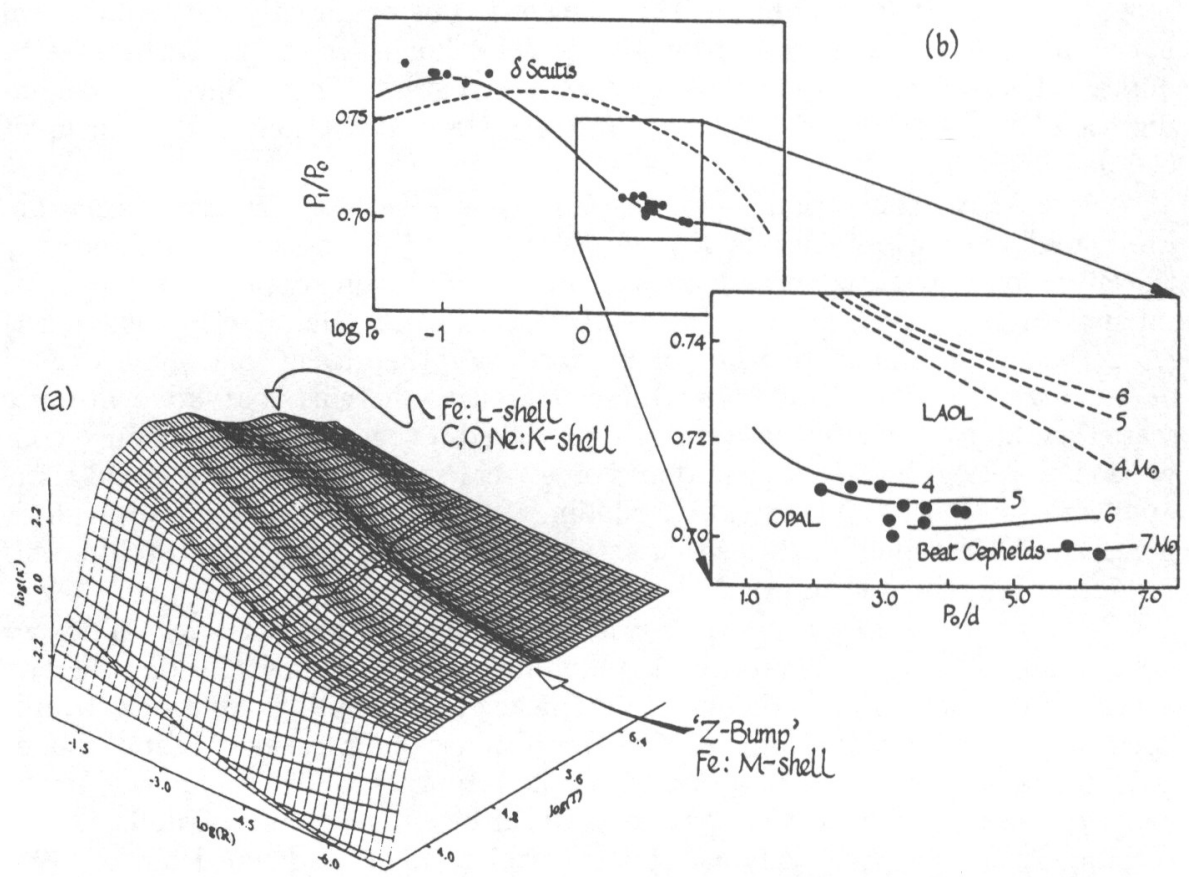

Figure 2. (a) The "opacity mountain" as obtained from OP calculations for a $\mathrm{X}=0.7, \mathrm{Z}=0.02$ composition. The two most prominent new features (ridges) found in the new opacity data are indicated. The solid line meandering along the slope of the $\kappa$-mountain marks the path traced out by the interior of a $12 M_{\odot}$ ZAMS model. (b) Petersen diagram $\left(P_{1} / P_{0}\right.$ vs. $\left.P_{0}\right)$ for population I variables $(\delta$ Sct and double-mode Cepheids). Dots indicate observed period data. Dashed lines clearly display the disagreement with the old LAOL data. Solid lines show the results of Moskalik et al. (1992) (lower right) and of ChristensenDalsgaard (1993)(upper left), both based on OPAL opacity data.

\section{Applications to classes of pulsating variables}

Without doubt, the new generation of stellar Rosseland-mean opacity data boosted considerably the activities in the computational branch of the stellar pulsation community. Accounting for $\Delta n=0$ transitions in the atomic calculations increased the Rosseland means by up to a factor of 3 or 4 (depending on the density range) at temperatures around $200000 \mathrm{~K}$. This bump in the opacity is often referred to as the "Z-bump" since it is caused mainly by inter - M-shell transitions of $\mathrm{Fe}$; but also more subtle contributions from $\mathrm{Ni}, \mathrm{Mn}$, and $\mathrm{Cr}$ are seen (Seaton et al. 1994). New stellar opacities are now available from two independently working groups using also different approaches to the microphysics: OPAL (Rogers \& Iglesias 1992; Igelsias et al. 1992) and OP (Seaton et al. 1994). Comparisons of the results of these two groups are very encouraging. 
A second, smaller bump - with an increase of the Rosseland mean by about $10 \%$ over the old LAOL data - is found around $10^{6} \mathrm{~K}$. In contrast to the Z-bump, this hotter bump , essentially caused by L-shell transition in Fe and by K-shell transitions in $\mathrm{C}, \mathrm{O}, \mathrm{Ne}$, is "seen" by some solar oscillation modes. The envelope convection zone stops at temperatures below that of the hotter bump so that the sound speed is modified by its presence. The new opacities were identified as an important contributor to an improved solar "standard model" (Guenther et al. 1992).

For the period ratios of the different radial overtones of Cepheids and other variables in the classical instability strip, it was the Z-bump that had a major impact. The mass discrepancy for the double-mode and for the bump Cepheids is now considered to be essentially resolved (Moskalik et al. 1992). Also the RR Lyrae variables - despite their having Population II heavy element admixtures - showed modified period ratios. The combination of an improved treatment of hydrogen in a non-ideal stellar plasma - leading to a reduced opacity below $\approx 100000 \mathrm{~K}$ - together with the reduced Z-bump are considered to be responsible for the particular behaviour. In contrast to the Cepheids, the new RR Lyrae period ratios do not improve the situation. Calculations by Kovács et al. (1992a) reveal considerable disagreement between double-mode masses for both Oosterhoff-type double-mode RR Lyrae stars and canonical horizontal-branch stellar evolution theory. A possible solution coming from non-solar admixtures of elements (such as $\mathrm{C}, \mathrm{N}, \mathrm{O}, \mathrm{Na}, \mathrm{Mg}$ ) was suggested (Kovács et al. 1992b).

Double-mode pulsators - Cepheids as well as RR Lyrae stars - can still not be simulated in a convincing way. For RR Lyrae stars at least, numerical models pulsating simultaneously in two modes, with both of them being present for a long times, were obtained recently. Kovács \& Buchler (1992) mentioned that purely radiative models can reproduce correct, i.e., observed, period ratios but they do not match the observed amplitude ratios. It was also realized that viscosity - numerical or physical - plays a crucial role for the final outcome. Bono \& Stellingwerf (1993) announced their success in producing double-mode RR Lyrae models including convection. They noticed that their double mode models satisfy the resonance condition $3 \omega_{0}-\omega_{1}=\omega_{2}$ (with $\omega_{i} \equiv 1 / P_{i}$ ). On the other hand, Kovács \& Buchler (1988) observed a $2 \omega_{0}=\omega_{3}$ resonance to obtain for their double-mode candidates. If, however, resonances are really needed or play a crucial role for the explanation of the double-mode phenomenon remains to be shown.

The OPAL/OP tables had a another important impact on pulsation theory. The Z-bump causes pulsational driving for stars in favourable temperature and luminosity ranges. The instability region running more or less parallel to the upper main sequence was eventually identified as an iron instability strip. Hence, the basic destabilisation mechanism of the $\beta$ Cepheids is classical $\kappa$-mechanism. The Z-bump is found to drive one or two radial (fundamental and/or first overtone) and a few low-order, low-degree $p$-modes above roughly $8 M_{\odot}$. At the lowmass end of the $\beta$ Cep strip some additional unstable $g$-modes of high spherical degree and high radial order were found numerically (Dziembowski et al. 1993). The $g$-modes are excited by being trapped such that radiative dissipation in the deep interior is considerably reduced. For the unstable $p$-modes it is the classical argument (e.g., Cox 1980) of the correspondence of the thermal time-scale of the 
envelope overlying the driving region and of the pulsation period that obtains. A more detailed discussion of B-type pulsators is presented by Moskalik (these proceedings).

The driving and damping contributions to the total work integral are nearly equal for the B-star pulsators. Small changes in the form and the location of the Z-bump have, therefore, considerable influence on the position and on the extension of the instability region. The presence or absence of $\beta$ Cepheids in stellar systems are being considered as rough metallicity indicators. Furthermore, the Warsaw group found (see Moskalik, these proceedings) that the boundaries of the instability strip react also on the particular handling of $\mathrm{Ni}, \mathrm{Cr}, \mathrm{Mn}$ in the opacity calculations. It is the different treatment of these elements by the OP and the OPAL group that allows the pulsation theorists to investigate the influence of these subtle aspects on the pulsation properties of stars.

Towards lower masses, below about $7-8 M_{\odot}$, to about $3 M_{\odot}$ the slowly pulsating B (SPB) stars are observed having long periods between about 0.4 3 days. These stars are now understood as a natural extension of the pulsation mechanism of the $\beta$ Cepheids into the $g$-mode domain (Gautschy \& Saio 1993, Dziembowski et al. 1993). For cooler stars the characteristic thermal time-scale of the envelope lying above the driving region of the Z-bump increases and tends to destabilize longer periods, in this case this is equivalent with higher-order $g$ modes. At the low-mass boundary of the SPB instability region the radial orders of the overtones favoured for destabilisation are so high that radiative dissipation due to the short spatial length-scales of the eigenfunctions eventually wins over the Z-bump driving. For further discussion see also Moskalik (these proceedings). The essence of this explanation is that the SPB stars can, physically spoken, be understood in the same manner as the $\beta$ Cepheids. In this respect, they are the same.

Also massive-star models are influenced by the Z-bump. The question of the upper-mass limit for stable main sequence models was readdressed after the new opacities became available. Stothers (1992) claimed that for a $\mathrm{Z}=0.03$ sequence of stellar models, only models exceeding $148 M_{\odot}$ turned unstable by the $\epsilon$-mechanism. Glatzel \& Kiriakidis (1993), on the other hand, found that the fundamental radial mode became pulsationally overstable already at $79 M_{\odot}$. The contribution of the nuclear burning to the instability was, however, of minor importance. In the calculations of Glatzel \& Kiriakidis, the envelope was the main contributor to the driving. They identified the Z-bump to induce strange modes (for a discussion see Gautschy \& Glatzel 1990, Glatzel 1994) that are responsible for strong pulsational instabilities in the upper main sequence star models. Even down to $58 M_{\odot}$ models, strange mode instabilities were encountered at high frequencies intermingled in higher overtone regular pulsation modes. If pulsational instabilities have any influence on the structure and on the evolution of very massive stars, then the strange modes, and not nuclearly unstable modes, dictate the outcome.

Kiriakidis et al. (1993) analyzed a large number of stellar evolution models with respect to their pulsational properties. Very broad domains of pulsational instabilities due to strange modes (see Fig. 3) were encountered. As strange modes, induced by the Z-bump, dominate in the hot domain of the HR diagram, i.e., close to the main sequence for the massive stars, their instabilities 


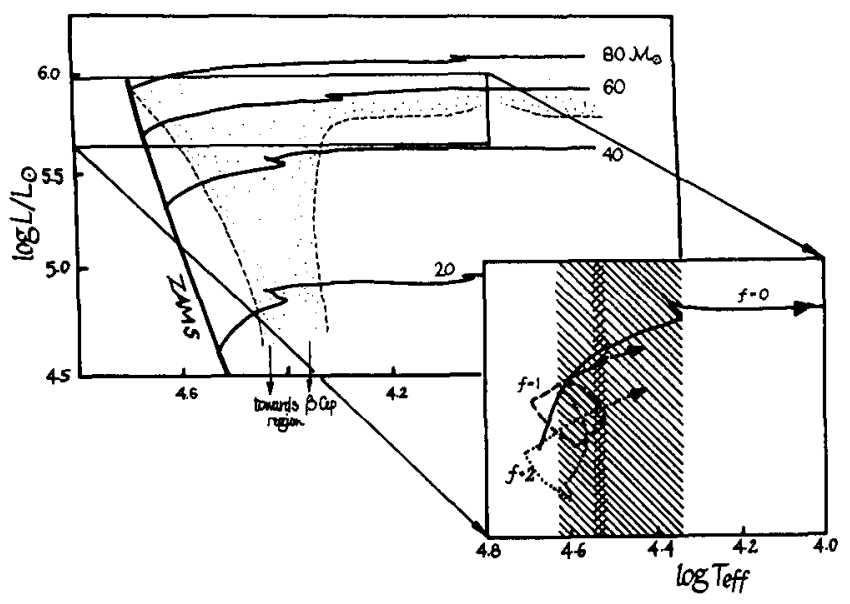

Figure 3. High luminosity part of the HR diagram. The evolutionary tracks indicate the stellar mass domain analyzed towards pulsational instabilities by Kiriakidis et al. (1993). The instability domain (for $Z=$ 0.02 is outlined by the dotted region. The inlet at the lower right shows stellar evolution calculations accounting for mass-loss parameterized by the growth rates of the pulsations (Langer et al. 1994). Details are given in the text.

are again sensitive to the heavy element abundances. Kiriakidis et al. (1993) claimed that they might have uncovered the origin of the Humphreys - Davidson limit (Humphreys \& Davidson 1984). Langer et al. (1994) used the results of the pulsation analyses of Kiriakidis et al. (1993) for their massive-star evolution calculations. The basic new hypothesis was that the pulsations induce mass loss that can be parameterized by the unusually large growth rates of the most unstable mode. The simplified parameterization introduced by Langer et al. was calibrated with the result of one particular non-linear simulation of Kiriakidis (1992). The result of the stellar evolution, assuming that the pulsations themselves do not react on the strong mass loss, are shown in the inset at the lower right of Fig. 3. The different values of $f$ indicate the effect a multiplicative factor in the mass-loss rate formula on the evolutionary track. The assumption that the unstable pulsation modes do not react on the modified envelope structure due to the substantial mass loss is certainly too naive. Nevertheless, the experiment of Langer et al. (1994) is a first step in the right direction of coupling pulsation properties with stellar evolution.

Upon the rumour of short-period pulsations in Wolf-Rayet stars (Blecha et al. 1992), Glatzel et al. (1993) investigated the stability properties of helium main-sequence models. Such models were considered reasonable approximations to observed Wolf-Rayet stars. Glatzel et al. (1993) were confronted, as already before in the case of hydrogen main-sequence stars, with pulsational instabilities due to strange modes at masses above $4 M_{\odot}$. The periods of unstable modes ranged from about one to 25 minutes. The pulsation mode destabilised by $\epsilon$ mechanism at masses above $14 M_{\odot}$ has a period of about 30 minutes, in contrast. 
Again, the growth rates of the unstable strange modes were orders of magnitude higher than the weak instability of the $\epsilon$-destabilised mode.

Some subclasses of hydrogen-deficient stars - very luminous, low-mass stars are known to be pulsating. In recent years it was realized that the very broad instability domain of these stars can be understood with unstable strange modes (e.g., Gautschy \& Glatzel 1990). A few stars in this peculiar class of pulsators defied an explanation, however. One of them is V645 Her, the low luminosity was insufficient to induce unstable strange modes in its envelope. Applying the new opacity data, Saio (1993) was able to show that this star (and the same applies for LSS 3184) are pulsationally destabilised by the Z-bump in the same manner as the $\beta$ Cepheids. Hence, instability mechanism for the low luminosity H-deficient stars is different from that of the luminous ones. Saio found the iron strip to extend and widen towards high luminosities and eventually to merge with the very broad instability domain of the strange modes.

Based on the results for H-deficient stars, Gautschy (1993) studied postAGB stars that are suitable candidates for cool central stars of planetary nebulae (CSPNe), with regard to possible pulsational instabilities. For masses exceeding roughly $0.75 M_{\odot}$, unstable strange modes could indeed be identified again. The coolest CSPNe are predicted to show periods around half a day with sharply decreasing periods as the stars get hotter. Very similar results were obtained for Wolf-Rayet - type CSPNe with their peculiar chemical abundances (Gautschy 1995). Observations, in particular newly available data from old Sonneberg monitoring plates, confirmed that the peculiar star FG Sge was pulsating already in the 1930s (Van Genderen \& Gautschy 1995). This supports the theoretical findings of very broad pulsational instability domains in the upper HR diagram for stars with high $L / M$ ratios.

In the domain of oscillating white dwarfs the WET consortium in particular continues to challenge stellar pulsation theory (see the contributions of Kawaler and Bradley in these Proceedings). In their approach, all conclusions drawn from the high-resolution oscillation-frequency spectra are based on the framework of adiabatic pulsation theory. Besides the limitations of perturbation approaches to account for rotation and magnetic fields, adiabatic theory is probably on safe grounds. Presently, the main goal is to understand and to self-consistently embed the "seismological" results in the theory of stellar structure and evolution.

Nonadiabatic theory, on the other hand, is less well understood. For seismological work this is, however, not crucial. Nevertheless, it is important to understand the physical mechanism behind destabilizing white dwarf oscillation modes. We will content ourselves here with considering the family of DA variables only.

For more than a decade, the $\mathrm{ZZ}$ Ceti variables were advocated to admit thin $\mathrm{H}$ envelopes with $M_{\mathrm{H}} / M_{*}<10^{-8}$ only (see e.g., Bradley et al. 1989, and references therein). White dwarf models with thicker envelopes always turned out to be pulsationally stable in the numerical calculations. Only very recently, with more careful modeling and more sophisticated computer codes, it was recognized (Bradley \& Winget 1994, Fontaine et al. 1994) that the stability properties of DAVs are actually rather insensitive to the hydrogen layer thickness, at least for $\mathrm{H}$ envelope masses below $10^{-4} M_{*}$. Furthermore, mode identification in variable DA white dwarfs was hinting at thick hydrogen envelopes (Fontaine et al. 
1992 for G226-29, Bergeron et al. 1993 for GD 165 and the review by Clemens 1993) for some time. Members of the stellar evolution community are certainly delighted about these recent developments. For the first time the results from oscillation theory of DAVs are consistent with predictions from canonical stellar evolution theory concerning the expected amount of hydrogen-rich material floating on top of white dwarfs. It is the nuclear burning during the pre white-dwarf evolution that essentially fixes that thickness. Very thin envelope hydrogen layers could be explained only if significant mass loss or some mixing mechanism could be identified.

The extension of the convection zone in $\mathrm{ZZ}$ Ceti variables is another source of uncertainty. This aspect is important for the understanding of the $g$-mode instability since convection - pulsation interaction seems to play a yet incompletely understood role. To match the theoretical ZZ Ceti blue edge with observed temperatures of the hottest ZZ Ceti star (the present candidate is G117-B15A), the efficiency - and hence the mixing-length - of the convection in the outermost layers of the DAVs is adjusted in an ad hoc way. The spectroscopic calibration of these white dwarfs is also far from trivial and hence far from unanimously accepted. Estimates of $\mathrm{T}_{\text {eff }}$ for G117-B15A, and hence of the ZZ Cetis' blue edge, decreased by roughly $800 \mathrm{~K}$ within the last decade (Weidemann \& Koester 1984, Koester et al. 1995). In any case, pulsation theorists postulated rather efficient and hence thick convection zones, based on modified mixing-length approaches, to comply with the observed blue boundary of the instability domain. Recent results from 2-D radiation hydrodynamic simulations (Ludwig et al. 1994) do not support the view of deep convection zones. Heuristic fits of mixing-length models to the full dynamical simulations seem to favour a mixing length of 1.5 pressure scale heights. This applies at least to the locations of vanishing entropy gradients. The thermal structure of the hydrodynamically simulated convection zone, however, differs considerably from one based on a mixing-length approach. Obviously, even the simplest class of pulsating white dwarfs might require some more work before we can claim with certainty to have understood them.

Acknowledgments. Financial support from the Swiss National Science Foundation and from the IAU is gratefully acknowledged.

\section{References}

Balmforth, N.J. 1992a, MNRAS, 255, 603

Balmforth, N.J. 1992b, MNRAS, 255, 632

Balmforth, N.J. 1992c, MNRAS, 255, 639

Bergeron, P., et al. 1993, AJ, 106, 1987

Blecha, A., Schaller, G., \& Maeder, A. 1992, Nature, 360, 320

Bono G.,\& Stellingwerf, R.F. 1993 in IAU Colloq. 139, New Perspectives on Stellar Pulsation and Pulsating Variable Stars, eds. J. M. Nemec \& J. M. Matthews, (Cambridge:University Press), p. 275

Bradley, P.A., \& Winget, D. E. 1994, ApJ, 421, 236

Bradley, P.A., Winget, D.E., \& Wood, M.A. 1989, in IAU Colloq. 114, White Dwarfs, ed. G. Wegner (New York:Springer), p.286 
Brown, T.M., et al. 1994, ApJ, 427, 1013

Castor, J.I., Davies, C.G., Davison, D.K. 1977, Los Alamos Rep. LA-6664

Clemens, J.C. 1993, Baltic Astronomy, 2, 467

Cox, J.P. 1980, Theory of Stellar Pulsation, (New Jersey:Princeton Press)

Christensen-Dalsgaard, J. 1993, in IAU Colloq. 137, Inside the Stars, eds. W.W. Weiss \& A. Baglin, (ASP: San Francisco), p. 483

Cutler, C. 1991, ApJ, 374, 248

Cutler, C., \& Lindblom, L. 1992, ApJ, 385, 630

Dziembowski, W.A., \& Goode, P.R. 1992, ApJ, 394, 670

Dziembowski, W.A., Moskalik P., Pamyatnykh, A.A. 1993, MNRAS, 265, 588

Feuchtinger, M., Dorfi, E.A. 1994, A\&A, 291, 209

Fontaine, G., Brassard, P., \& Wesemael F. 1994, ApJ, 428, L61

Fontaine, G., Brassard, P., Bergeron, P., \& Wesemael, F. 1992, ApJ, 399, L91

Gautschy, A. 1993, MNRAS, 265, 340

Gautschy, A. 1995, A\&A, in press

Gautschy, A., \& Glatzel, W. 1990, MNRAS, 245, 597

Gautschy, A., \& Saio, H. 1993, MNRAS, 262, 213

Gehmyer, M. 1992, ApJ, 399, 265

Gehmyer, M. 1993, ApJ, 412, 341

Van Genderen, A., \& Gautschy, A. 1995, A\&A, 294, 453

Glatzel, W. 1994, MNRAS, 271, 66

Glatzel, W., \& Kiriakidis M. 1993, MNRAS, 262, 85

Glatzel, W., Kiriakidis M., \& Fricke K.J. 1993, MNRAS, 262, L7

Goode, P.R., \& Thompson, M.J. 1992, ApJ, 395, 307

Gough, D.O., \& Thompson, M.J. 1992, MNRAS, 242, 25

Goupil M.-J., \& Buchler J.R. 1994, A\&A, 291, 481

Guenther, D.B., Demarque, P., Kim, Y.-C., \& Pinsonneault, M.H. 1992, ApJ, 387,372

Van Hoolst, T. 1994, A\&A, 292, 471

Humphreys, R.M., \& Davidson, K. 1984, Science, 223, 243

Iglesias, C.A., Rogers, F.J., \& Wilson, B.G. 1992, ApJ, 397, 717

Ipser, J.R., \& Lindblom L. 1990, ApJ, 355, 226

Kiriakidis, M. 1992, $\mathrm{PhD}$ thesis, Göttingen

Kiriakidis, M., Fricke, K.J., \& Glatzel, W. 1993, MNRAS, 264, 50

Koester, D., Allard, N., \& Vauclair G. 1995, A\&A, in press

Kokkotas, K.D., Schutz B.F. 1992, MNRAS, 255, 119

Kovács, G., \& Buchler, J.R. 1988, ApJ324, 1026

Kovács, G., \& Buchler, J.R. 1992, ApJ, 404, 765

Kovács, G., Buchler, J.R., \& Marom 1992a, A\&A, 252, L27

Kovács, G., Buchler, J.R., Marom, A., Iglesias, C.A., \& Rogers, F.J. 1992b, A\&A, 259, L46 
Kurtz, D.W., Kanaan, A., \& Martinez, P. 1993, MNRAS, 260, 343

Langer, N., Hammann, W.-R., Lennon, M., Najarro F., Pauldrach, A.W.A., \& Puls, J. 1994, A\&A, 290, 819

Ludwig, H.-G., Jordan, S., \& Steffen, M. 1992, A\&A, 284, 105

Moskalik, P., Buchler, J.R., \& Marom, A. 1992, ApJ, 385, 685

Rogers, F.J.,\& Iglesias, C.A. 1992, ApJS, 79, 507

Saio, H. 1993, MNRAS, 260, 465

Seaton, M.J., Yu Yan, Mihalas D., \& Pradhan, A.K. 1994, MNRAS, 266, 805

Stellingwerf, R.,F. 1984, ApJ, 284, 712

Stothers, R.B. 1992, ApJ, 392, 706

Takata, M., \& Shibahashi, H. 1994, PASJ, 46, 301

Weidemann, V., \& Koester D. 1984, A\&A, 132, 195

Xiong, D.R. 1981, Scientia Sinica, 24, 1406

Xiong, D.R. 1991, Proc. ASA, 9, 26

\section{Discussion}

Kawaler: Are you sure that the $d s / d r<0$ line for the hydrodynamic models of white dwarf envelopes is correct? It is within the photosphere $\left(\sim 10^{-16} M_{\odot}\right)$ and looks to be where the top of the convection zone is in the interior models of Tassoul et al. (1990). Also, where was the bottom of the hydrodynamic model grid within the stellar model?

Gautschy: The line is correct. Indeed, the disagreement in depth is rather frightening. The bottom of the computational box of the hydrodynamic simulations lies at least at about $10^{-13} \mathrm{M}_{*}$.

Christensen-Dalsgaard: Have you considered using the Gough-Balmforth theory for time-dependent convection in the white-dwarf case? It appears to work quite well in the Sun and solar-like stars.

Gautschy: If you give me more time to work on, then I certainly will try to include a nonlocal time-dependent treatment in the oscillation code.

Goldreich: Don't you have a big problem handling dynamic convection when you calculate growth or decay rates for $g$-modes of white dwarfs?

Gautschy: The full treatment of convection dynamics and its interaction with the oscillation is at the moment out of reach. It should be included particularly when the convection zone is deep. If it turns out to be shallow, convection might adapt to the particular pulsation phase on times shorter than the period of the pulsation. The state at the moment is that I just did the the simplest thing and it did not work. 